\title{
DIVERSITY OF PHOTOGRAMMETRIC APPROACHES FOR MULTI-PURPOSE APPLICATIONS
}

\author{
Aleksandra Bujakiewicz ${ }^{1}$, Jakub Markiewicz ${ }^{2}$, Krzysztof Bakuła ${ }^{2}$, \\ Dorota Zawieska $^{2}$ \\ 1) Koszalin University of Technology \\ Faculty of Civil Engineering, Environmental and Geodetic Sciences, \\ Koszalin, Poland \\ 2) Warsaw University of Technology \\ Faculty of Geodesy and Cartography, Warsaw, Poland
}

\begin{abstract}
The presented paper shows the diversity of photogrammetric approaches by introducing a problem of camera interior orientation, data georeferencing, data integration and the issue of automatic object surface modelling. The authors discuss the abovementioned issues on the example of the work carried out at the Faculty of Geodesy and Cartography, Warsaw University of Technology referring to solutions practiced in Computer Vision. This article is an example of introduction of photogrammetry as a discipline open to achievements of close fields of science, which is widely applied in practice.
\end{abstract}

Keywords: photogrammetry, computer vision, image orientation, data integration, image matching

\section{Introduction}

Photogrammetry has a very long history over one hundred years, and therefore, its development had many stages related to general technological advancements. During the last 20 years, digital photogrammetry, which was permanently developing, opened many new opportunities for its traditional and new applications. There is a variety of non-contact imaging techniques to record reliable information about the Earth surface and various physical objects. The traditional imagery data from multi sensors; such as aerial and terrestrial digital cameras, scanning systems and VHRS satellite imagery, are usually utilized. In addition, such systems as Unmanned Aerial Vehicles (UAV), Terrestrial Mapping Mobile Systems (MMS), motion imagery systems, are becoming more popular. 
High accuracy of the photogrammetric measurements usually requires a very precise sensors calibration and also very reliable data geo-referencing, either during imagery acquisition with use of high performance GPS/INS system or in postprocessing stage. A complexity of the photogrammetric products, often requires combining various types of source data, such as airborne and terrestrial scanning and images data, in this case their inter-calibration is expected. Such a variety of imagery data requires diversity of approaches for their processing and needs vast knowledge and mobilization of the researchers to be involved in the development of methodology for various applications.

In presentation on 'Developments in Photogrammetry', Steward Walker (2012), the invited speaker of XXII ISPRS Congress in Melbourne, exposed the exponential growth in data capture, mainly high resolution imagery, UAVs, social media, community based mapping with a challenge of fusion, especially in real time. He also emphasized the understanding of the importance of location in decision making and the use of geospatial data by non-experts; availability of metadata anywhere, anytime as well as in real- time. He concluded that photogrammetry is an advanced science with GIS and remote sensing convergence, with automation of conventional production lines and increasing number of applications.

Konrad Schindler, the second invited speaker of ISPRS Congress 2012, in his presentation (2012) on 'Developments in Computer Vision and its Relation to Photogrammetry' exposed strong convergence between $\mathrm{CV}$ and $\mathrm{PH}$, saying that they are not distant cousins but sister sciences. Comparing the definitions of both, he noticed, that they are of similar meaning, but formulated in a different way. It is, however, emphasized that there are some differences, since developments in $\mathrm{PH}$ are application-driven whereas in CV they are methods-driven. Photogrammetry is involved in many more applications, topographic and close range, than computer vision, but unfortunately $\mathrm{PH}$ has about six time less money and specialists than $\mathrm{CV}$. It is also underlined by Schindler that selected developments in $\mathrm{CV}$ in the last few years are potentially relevant to $\mathrm{PH}$, especially in the areas of semantic image understanding, automate object recognition, detection and extraction (also from point clouds); tracking, dynamic processes from image sequences and physics-based 3D reconstruction.

Over twenty years the relations and differences between these two disciplines have been discussed. In a paper by Mundy (1993), the root of the problem between $\mathrm{PH}$ and $\mathrm{CV}$ communities were raised. It has been exposed, that $\mathrm{PH}$ is a mature subject with a well established problem descriptions and solutions, and $\mathrm{PH}$ community is generally not very tolerant for the results couched in alternative terminology and with somewhat different goals. Many results developed by CV researchers had been already known much earlier by $\mathrm{PH}$ and existing photogrammetric theory still has much to offer to the problems of object recognition and scene modelling. At the same time, results from CV can help to advance photogrammetry both in the discovery of completely new principles for methods as well as the automation of complex feature extraction. Mundy has addressed a few key questions concerning the goals of $\mathrm{CV}$ and $\mathrm{PH}$. In $\mathrm{CV}$ three major technical goals are exposed: object recognition algorithms to operate at the same class for an object defined by the human conceptual framework; vehicle navigation to provide guidance to an autonomous vehicle along the defined path and at the same time to avoid 3D structures; automatic object modelling to recover a complete and reasonable accurate model of an object with minimum human intervention. In $\mathrm{PH}$, the major goal is a high accuracy, which is required in a wide range of applications, topographic and 
large scale mapping (aerial and VHRS) and also close range, which includes also automatic object modelling. Very comprehensive panel discussion on a dialog between CV and PH was conducted by Forstner (2003). Some technical questions from $\mathrm{CV}$ to $\mathrm{PH}$ were raised, such as, why $\mathrm{PH}$ is only interested in the highest precision, use the calibrated cameras, or have an inadequate level of automation. The questions raised from $\mathrm{PH}$ to $\mathrm{CV}$ were opposite. $\mathrm{PH}$ asked $\mathrm{CV}$ why they do not care about precision, use only un-calibrated cameras, have only interest in theoretical results. Some of the questions have showed, that both $\mathrm{PH}$ and $\mathrm{CV}$ had no proper knowledge what the others are doing. It has been changing slowly by the involvement of both $\mathrm{PH}$ and $\mathrm{CV}$ specialists in common projects and conferences. As it can be seen from many publications, both, metric and non- metric imageries and self calibration approaches, have been applied by $\mathrm{PH}$ since many years. In some $\mathrm{PH}$ experiments, executed for example by Mayer et al. (2003) and Zawieska (2012), the approaches for fully automatic calibration, orientation of images sequence and 3D reconstruction, were adopted from CV. They are based on triplets, where the trifocal tensor comprises a linear means for the description of the relation of three perspective images.

In the following chapters, some experiments performed in the Department of Photogrammetry and Remote Sensing, Warsaw University of Technology, will be presented. They show a diversity of photogrammetric approaches in three areas: interior orientation and georeferencing of imaging data, data integration (particularly laser scanning and imagery) for objects reconstruction and automatic object surface modelling.

\section{Interior orientation and georeferencing of imaging data}

In most photogrammetric applications accuracy is the major priority. To satisfy this requirement the parameters of interior and exterior orientation of the source data have to be precisely determined. In general, the interior orientation parameters, which describe a shape of projective rays bundle, are determined in calibration process, either a'priori of imagery data acquisition, or during the post-processing stage (self calibration). A'priori methods of calibration depend on the type of imagery system. They can be executed either with a laboratory equipment (goniometer) or with use of different calibration tests. For aerial metric cameras (used for topographic and large scale mapping) and also for terrestrial metric and non-metric cameras of close range, various types of calibration tests are applied. Methods for calibration of non - metric cameras have been developed for many years, using different mathematical models for correction of the image systematic errors effect. The specific case of calibration process is when a camera is used for taking images in a very large scale. Such case was applied in departmental research project (Bujakiewicz et al., 2006b), where the calibration procedure was based on 3D and 2D chess-board calibration tests (Fig. 1.).

In such a solution enabled by multi-image triangulation (aerial or terrestrial) called self-calibration, which is the most general approach, all interior orientation parameters, with effect of the image systematic errors and with exterior orientation of all images are determined in the same adjustment procedure. When the acquisition system includes a few imagery subsystems, as for example, a few cameras and a laser scanner, together with a navigation system GPS/INC, mounted on the same platform (as in a MMS system), then all linear and angular excentrics have to be determined in an inter-calibration procedure. 


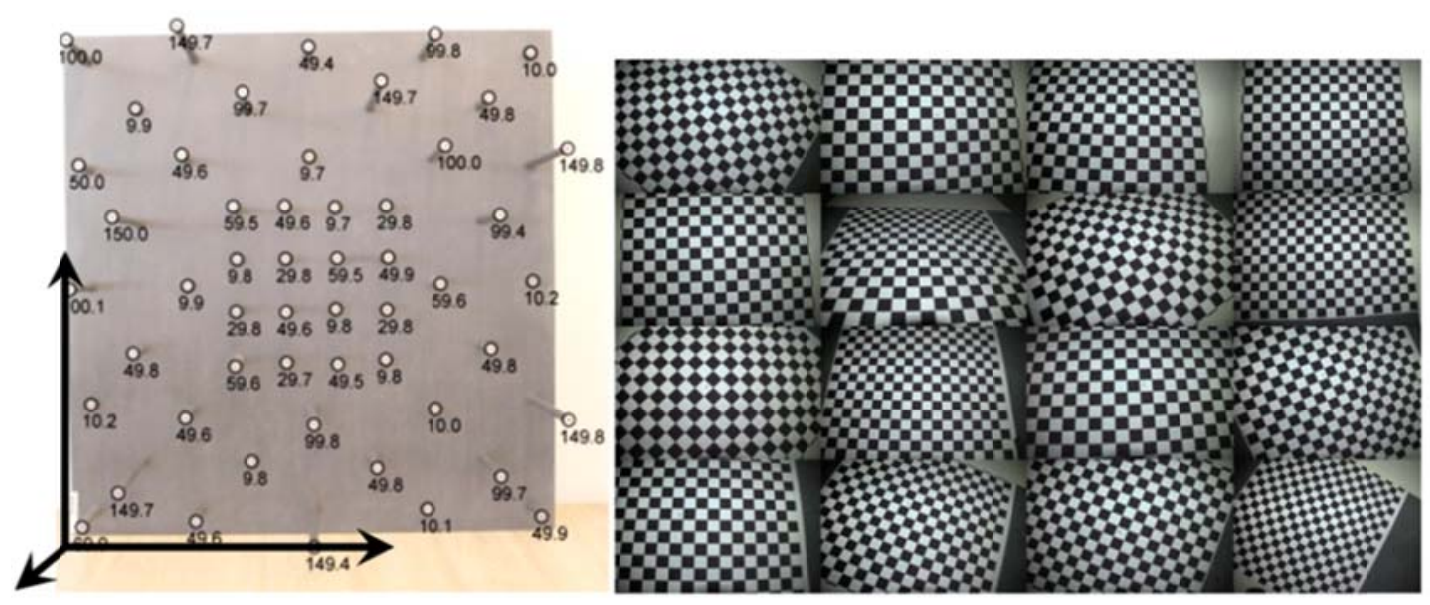

Fig. 1. Calibration tests $3 \mathrm{D}$ and $2 \mathrm{D}$ for very large close range photographs (Bujakiewicz et al., 2006b)

In any type of photogrammetric compilation, all acquired imagery data have to be oriented in respect to the reference coordinate system, either during the acquisition process (on-flight), called direct georeferencing, or in the post- processing stage indirect georeferencing. Direct georeferencing of imagery data requires the imaging systems to be supported with the precise, very strictly integrated GPS and INS devices to provide the highly accurate exterior orientation of all collected data. When such devices accelerate orientation parameters of lower precision, then they are assumed as the approximate values in the post-processing. There are many approaches of the post-processing georeferencing (Bujakiewicz et al., 2011). The most general indirect georeferencing solution refers to the triangulation adjustment of multi-bundles (aerial or terrestrial). As the result, the exterior orientation parameters of all images in a block are simultaneously determined. Such an approach is used in most aerial mappings or close range applications. Such a case is shown in the project carried out for architectural inventory of the cathedral church in Radom, Poland (Lisowska, 2007). For the whole object, 80 digital photographs were taken with camera Hasselblad 905SWC. Simultaneous adjustment process of all observations with BINGO-F program accelerated the RMS of residuals for control points $0.007 \mathrm{~m}, 0.008 \mathrm{~m}, 0.006 \mathrm{~m}$ and check points $0.020 \mathrm{~m}, 0.013 \mathrm{~m}, 0.048 \mathrm{~m}$, for $\mathrm{X}, \mathrm{Y}$ and $\mathrm{Z}$, respectively.

The approach of multi-image bundle adjustment for a set of photographs with different pixel dimension and scale as well as with non-conventional overlap was applied to determine the exterior orientation parameters for six photographs collected for the altar in Warsaw church (Bujakiewicz et al., 2008). Two of stereo photographs, covering the entire altar, were taken by the metric camera at scale of about 1:200 and four images, covering two symmetrical parts of the altar, were taken with a nonmetric camera at scale about 1:40, with $10 \mu \mathrm{m}$ pixel size. After simultaneous adjustment of all observations, with the Orient/Orpheus program, the accuracy from 0.4 to 0.6 of the pixel size, depending on classes of identified points and imaging scale, were obtained.

Creation of data bases for different architectural monuments and engineering structures became very popular. Developments in the process of data acquisition by various close range photogrammetric systems, such as terrestrial scanning and metric or non-metric cameras, generate a wide spectrum of integration for both 
acquisition techniques, in order to obtain better results for geometrical accuracy, visualization and reconstruction efficiency of 3D models (Alshawabkeh \& Haala, 2004). A lot of attention was invested to demonstrate the complementing aspect of both acquisition techniques instead of vaguely and artificially emphasized competition case (Guarnieri et al., 2006). In order to integrate scanning and imagery data common georeferencing, in respect to the selected reference coordinate system, is required. When the scan and images are acquired for the object covered by some targeted control points, with known coordinates in the reference system (global or local), the exterior orientation of both data sets can be determined in one processing stage. Georeferencing of imagery data, useful in process of reconstruction of $3 \mathrm{D}$ realistic model from scanning and imagery data, can also be executed by use of some natural points identified within 3D scan model and measured on all photographs. The example of such approach is shown by Dominik (2008), where 4 scans from the IMAGER $5006(Z+F)$ and 20 non-metric images, taken with the Canon EOS 350D, were collected for reconstruction of 3D model of the fragment of Main Aula at Warsaw University of Technology (WUT). The exterior orientation parameters (together with corrections to interior orientation data) of all photographs were determined by the use of self-calibration multi-image exterior orientation module of Orient/Orpheus software. The accuracy of $2.7 \mathrm{~mm}$ in object space was reached. Similar approach for georeferencing was applied by Bakuła (2010). The final 3D realistic models of both objects are shown in figure $2 \mathrm{a}, \mathrm{b}$.

(a)

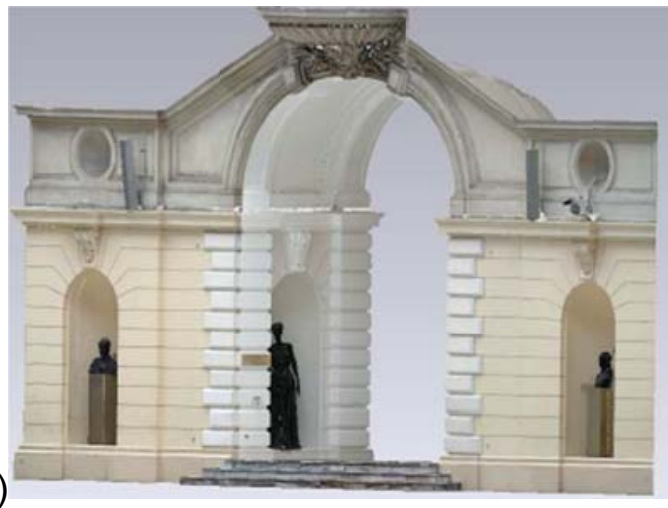

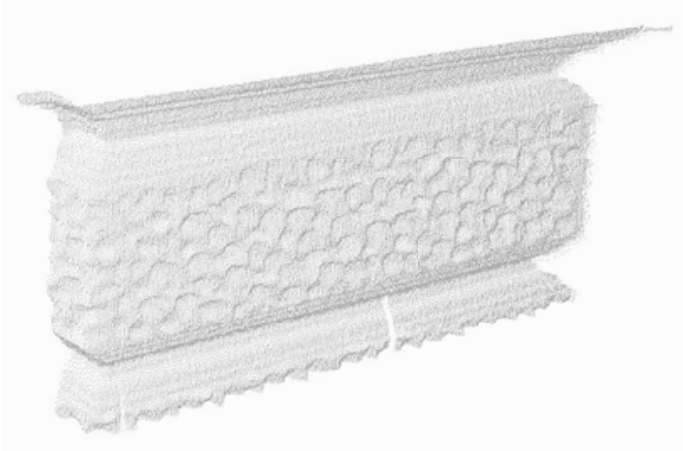

(b)

Fig. 2. The final $3 D$ realistic models from scanner and images data (a) part of Warsaw University of Technology Aula (Dominik, 20108) and (b) fragment of WUT façade (Bakuła, 2010)

Another approach refers to georeferencing of close range images taken for small 3D objects, architectural, archeological or mechanical details (Bujakiewicz et al., 2006a; Zawieska et al., 2012). In these cases, the objects are located within the special frame, equipped with control points of different spatial distribution and submillimeter accuracy. The example of such a frame with a set of 18 control points (built within the project undertaken at WUT) and two types of objects is shown in figure 3.a,b. 
(a)

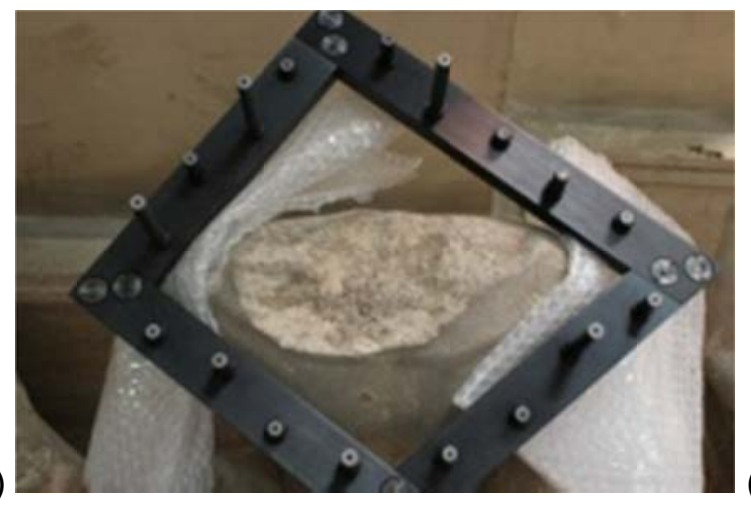

(b)

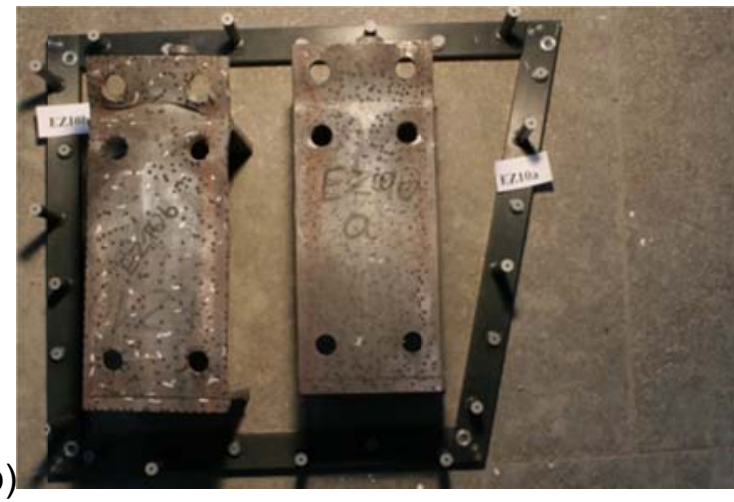

Fig.3. One of object stereo images within the control points frame:

(a) the archeological object from the Cairo Museum (Bujakiewicz et al., 2006a),

(b) the metal plate sample (Zawieska et al., 2012)

\section{Data integration}

As mentioned in Chapter 1, photogrammetry allows integrating the geospatial data which is understood as a combination of both data of the same measurement type and a synergy of different types of measurements in order to obtain additional information helping to improve the quality and the accuracy of the resulting 3D model. The integration of the same type of photogrammetric measurements (e.g. a position of the point) but obtained from various data sources is particularly related to the improvement and supplement some of the technologies disadvantages (Kurczyński, 2006). Other purpose of such integration is to obtain more detailed and more accurate three-dimensional models that can be collected in detailed analyzes during research on shape and geometric relationships (Briese et al., 2003). The use of various types of information, for example, the location determination and intensity registration of laser reflections and color assignment allow a deeper analysis of the collected data, giving a wider opportunity for their interpretation. This chapter presents the data subjected to an integration process in the selected photogrammetric projects, as well as its influence on the final data.

The photogrammetric approach used for the development of three-dimensional models is typically based on various data sources, which are derived from terrestrial, airborne and satellite systems. Each of them has different range and possible accuracy and generate data for different purposes. Their integration can be useful in some cases. The range of registered area related to distance to object is one of the reasons for photogrammetric data integration. Other reasons of such operation are different technologies for data acquisition and various accuracy requirements. They can be lower for entire object modelling and therefore, data source of lower precision is adequate, but for some details of this object higher precision data are required.

Considering the way and the methodology adopted for the integration of data from different photogrammetric sources, the following issues should be respected: the highest possible geometric accuracy, the ability of all details representation, the realistic effect of modelling, low cost of operation, versatility of software and efficiency of created model. The prioritization of the above-mentioned factors influences the type of methodology of data integration (Berladin et al., 2003).

One opportunity to integrate different data is to divide them in a hierarchical classes. This approach depends on the required level of details' representation and the accuracy of their acquisition. An example of using aerial and terrestrial images to create the overall model shape and application of laser scanning technique for its 
details modelling is shown in figure 4 . In such projects, the integration of aerial and ground-based data is on the stage of relative orientation which is a common limitation. It is caused by the specification of measurements and type of data recording system. In topographic photogrammetry the most frequent example of data integration is a relative orientation of airborne laser scanning data and aerial photographs. In applications of close-range photogrammetry, the terrestrial laser scanning data with low aerial photography (Aguilera et al., 2003) or the ground-based images (Markiewicz, 2012) are widely applied (fig. 4a,b).

(a)

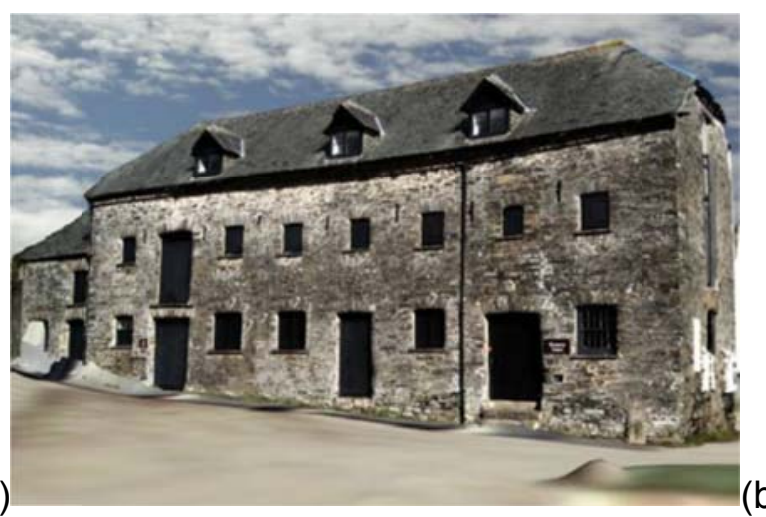

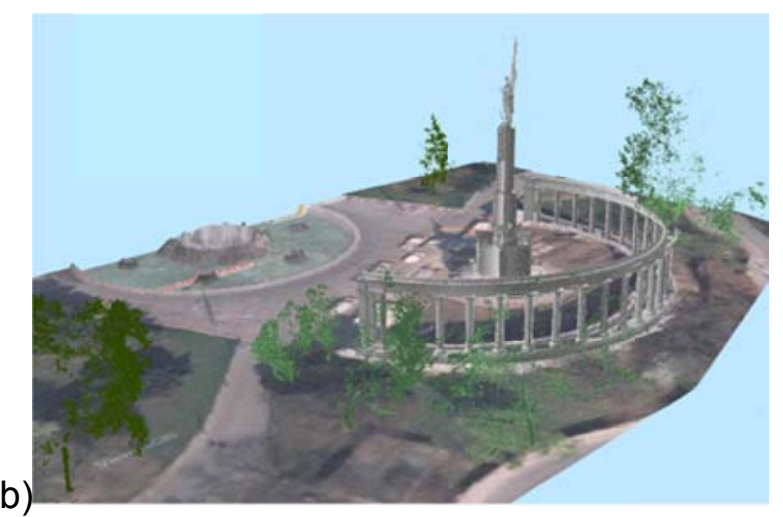

Fig. 4. Product of hierarchic data integration Aguilera et al, 2003 (a), and result of data integration in external non-photogrammetric software (Markiewicz, 2012) (b)

Considering the reasonableness of each data use, limitations that come with their use separately should be considered. It is a an example which demonstrates a complementarities of the two techniques. It should be noted, that airborne laser scanning measurements are based on sending and receiving laser beams in the near infrared spectrum (Nettley et al., 2011). The advantage of using such wavelength is the ability to penetrate and map vegetation. On the other hand, there is no possibility for acquisition of data corresponding to areas covered by water surface. Without an integration with image-based system, scanning has no opportunity to generate points cloud with texture information provided by RGB value assigned or data used for texturing digital surface models (DSM) (Krauss, 2007).

Terrestrial laser scanning might be subjected to the occurrence of dead areas in case of object glass measures. The possibility of reducing the influence of this problem is the integration with terrestrial digital images or increasing number of scanner positions (Briese et al., 2003 ). The principle of measurement and data collection is similar to the airborne laser scanning. Thereffore, during the measurement it is not possible to acquire additional information about RGB composition. In many engineering applications, this information is valuable, so this is a reason for combining the ground-based images and terrestrial laser scanning data.

\section{Automatic object surface reconstruction}

Contemporary close-range photogrammetric systems are based on direct acquisition of images, their calibration and geo-referencing (introduced in Chapter 2). In order to achieve the entire surface of a model, the continuous registration of successive images, with the sufficient overlap, is required. Such images are acquired with a digital photogrammetric camera (sequential images) or with a hand-held camera. 
Corresponding points on selected images are determined by 3D coordinates, as a result of appropriate crossings of projection rays.

A typical reconstruction in CV starts with the analysis of a pair of photographs (Pollefeys et al., 2004) or three photographs (Hao \& Mayer, 2003). Then the 'Structure \& Motion' (S\&M) operation is performed, in order to determine explicitly the camera stations and coordinates of object points. The unlimited number of photographs may be used for such calculations. According to the S\&M approach, the camera trajectory is automatically determined on the basis of several dozens or several hundreds of points, adjusted by means of a tracing algorithm (Brazetti \& Scaioni, 2009).

Photogrammetric generation of a digital surface model is a task, which always requires a set of points, characterised by the sufficient cardinality to be measured. Such a set is necessary to describe the shape of a measured object with the assumed accuracy. This task may be performed with the use of the digital, photogrammetric station and manual, semi-automatic or automatic photographs measurements. The flexible measuring environment, based on windows, allows for efficient transfer of points within the areas of multiple coverage. Automatic correlation and controlling indexes, calculated on the fly, increase the accuracy and speed of measurements. Automatic radiometric correlation supports implementation of measuring tasks by the operator. Automatic searching for corresponding points is performed using two methods: the first is based on matching intensity (Area Based Matching - $A B M$ ) and the second is based on feature matching (Feature Based Matching - FBM). The examples presented in figures $5 \mathrm{a}, \mathrm{b}$ illustrate results of surface generation with the use of those methods on photogrammetric workstations. The result of algorithms' combination applied in the CV has also been tested by Zawieska (2012). Selected operators for corner detection (detection of characteristic points), such as Harris, SUSAN, the ABM/LSM method and the tri-focal tensor have been used for automatic measurements and generation of surfaces shape of the selected objects (fig. $5 \mathrm{c}$ )

(a)

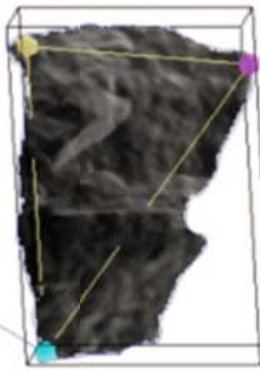

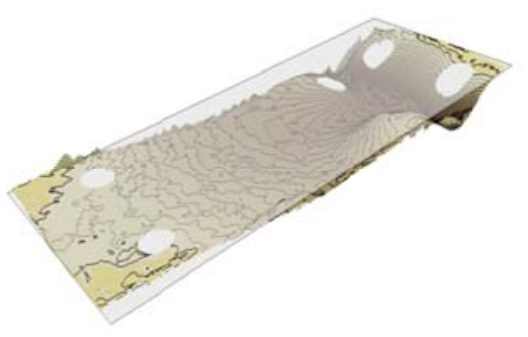

(b)

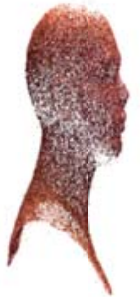

(c)

Fig. 5. 3D visualisation of surfaces: (a) fragment of the relief for the small archaeological sample (Bujakiewicz et al., 2006a), (b) surface of a metal plate (Zawieska et al., 2012), (c) a model of a mannequin's head (Zawieska, 2012).

In case of generation of 3D surface models of such small surfaces (dimensions of the order of $100 \times 400 \mathrm{~mm}$, difference in heights of the order $10 \times 20 \mathrm{~mm}$ ), the assumed accuracy of their measurements is of the order of $0.5 \mathrm{~mm}$. The accuracy of reference determination i.e. the control spatial co-ordinates should be ranged within the limits of $0.1 \mathrm{~mm}$, and the accuracy of matching the model into the control points within the limits of $0.3 \mathrm{~mm}$. In case of the presented examples the assumed accuracy has been met, i.e. the accuracy of surface reconstruction was from 0.3 to $0.8 \mathrm{~mm}$. 
In airborne photogrammetry also automatic surface reconstruction is used. Such products as digital terrain model and digital surface model, as well as 3D City models are generated by many organizations including governments (Kurczyński, 2006). In comparison to close-range applications such case is simpler approach because of use $2.5 \mathrm{D}$ system - only the $Z$ value is assigned to exact $X, Y$ coordinates, what makes it very simple and imposes utilization of regular GRID models. Such models are created automatically from airborne laser scanning data or they can be a result of the abovementioned aerial and satellite image matching.

\section{Conclusion}

Rapid development of terrestrial, aerial and satellite multi-sensors for imaging and inflight georeferencing, has been observed for last years. This has required an extensive research on new processing methods to generate and visualize the final photogrammetric products. In contrast to the issues under consideration of Computer Vision, photogrammetric technologies (both air and terrestrial) are focused on geospatial applications. Their role does not end with the visualization only, but it also includes the proper objects' reconstruction and their spatial orientation. Therefore, problems of rigorous sensors interior orientation on the basis of calibration data, as well as imagery data georeferencing (external orientation) in the selected reference system, are of primary importance. The integration of various, multi-sensorial data, which has come into use in some applications, is a complicated issue in photogrammetric solutions. In automated objects reconstruction, photogrammetry relies on classic and relatively simpler methods. However, rapid development of Computer Vision for object surface modelling affects the exchange of solutions used in some photogrammetric applications.

\section{References}

Aguilera, D., Lahoz, J., Finat, J., Martinez, J., Fernandez, J., \& San Josem, J. (2006). Terrestrial laser scanning and low-cost aerial photogrammetry in the archeological modeling of a Jewish tanneries. International Archives of Photogram., Remote Sensing, Volume XXXVIII-5/W1

Alshawabkeh,Y., \& Haala, N. (2004). Integration of Digital Photogrammetry and Laser Scanning for Heritage Documentation. International Archives of Photogrammetry and Remote Sensing, Volume XXXV, B5, Istambul

Bakuła, K. (2010). Comparison of the object shape generated from digital images and laser Scanning (MSc Diploma thesis), Warsaw University of Technology

Berladin, J.A., Picard M., El-Hakim J., Godin G., Bandiera A., \& Latouche D. (2003). Virtuayizing a Byzantine crypt by combining high-resolution textures with laser scanner 3D data. Proceedings of VMMS 202, pp. 3-14

Brazetti, L., \& Scaioni M. (2009). Automatic orientation of image sequences for 3D object reconstruction: first results of a method integrating photogrammetric and computer vision algorithms. International Archives of Photogram., Remote Sensing, Volume XXXVIII-5/W1 
Briese, Ch., Pfeifer, N., \& Haring, A. (2003). Laser scanning and photogrammetry for the modeling of the statue Marc Anton. Proceedings of XIX CIPA Symposium, Antalya

Bujakiewicz, A., Arcisz, M., \& Zawieska, D. (2008). Use of Multiscale Images for Data Bases of 3D Architectural Objects. Archives of Photogrammetry, Cartography and Remote Sensing,18a, pp. 39-48

Bujakiewicz, A., Kowalczyk, M., Podlasiak, P., \& Zawieska, D. (2006). 3D Reconstruction and Modeling of the Contact Surfaces for the Archeological Small Museum Pieces. International Archives of Photogrammetry and Remote Sensing, vol. XXXVI, Part 5, pp. 56-61

Bujakiewicz A., Kowalczyk M., Podlasiak P., \& Zawieska D. (2006). Calibration of Very Close Range Digital Cameras. Journal of PAN Geodesy and Cartography, 55, No. 2, pp. 95-108

Bujakiewicz, A., Podlasiak, P., \& Zawieska D. (2011). Georeferencing of Close Range Photogrammetric Data. Archives of Photogrammetry, Cartography and Remote Sensing, 23, pp. 91-104

Dominik, W. (2008). Generation of Realistic 3D Model from Terrestrial Laser Scanning and Imagery Data. (MSc Diploma thesis), Warsaw University of Technology

Forstner, W. (2002). Computer Vision and Photogrammetry - Mutual Questions: Geometry, Statistics and Cognition. Proceedings of. International Symposium of CIPA on Photogrammetry. meets Geoinformation, Potsdam

Guarnieri, A., Remondino F., \& Vettore A. (2006). Digital Photogrammetry and TLS Data Fusion Applied to Cultural Heritage 3D Modeling. International Archives of Photogram., Remote Sensing, Vol. XXXVI, Part 5

Hao, X., \& Mayer, H. (2003). Orientation and Auto-Calibration of Image Triplets and Sequences, IAPRSSIS, 34(3/W8), pp. 73-78

Kraus, K. (2007). Photogrammetry, vol.1, Fundamentals and Standard Processes, Bonn: Dümmlers

Kurczyński, Z. (2006). Aerial and satellite imagery of Earth. Warsaw: Warsaw University of Technology Publishing House

Lisowska, P. (2007). Use of Digital Photogrammetry for Architectural Inventory (Eng. Diploma thesis), Warsaw University of Technology

Markiewicz, J. (2012). Aspects of photogrammetric data integration for generation 3D models of the selected objects located in the urban space., Archives of Photogrammetry, Cartography and Remote Sensing, 24, pp. $199-209$ 
Mayer, H., Matthias, M., \& Peipe, J. (2003). Comparison of Photogrammetric and Computer Vision Techniques - 3D Reconstruction and Vizualization of Wartburg Castle. Proceedings of XIX CIPA Symposium, Antalya

Mundy, J.L. (1993). The Relationship between Photogrammetry and Computer Vision. Integration Photogrammetric Technologies., Scene Analyses and Machine Vision, Ed. SPIE, vol. 1944, pp. 92-105

Nettley, A., Anderson, K., DeSilvey C., \& Caseldine, C. (2011). Using terrestrial laser scanning and LiDAR data for photo-realistic visualization of climate impacts at heritage sites. International Archives of Photogrammetry, Remote Sensing and Spatial Information Sciences, vol. XXXVIII-5/W16,

Pollefeys, M., Van Gool, L., Vergauwen, M., Verbiest, F., Cornelis, K., Tops, J., \& Koch R. (2004). Visual modeling with a hand-held camera. IJCV, 59 (3), pp. 207232,

Schindler, K. (2012). Developments in Computer Vision and its Relation to Photogrammetry Invited paper of XXII ISPRS Congress in Malbourne

Walker, S. (2012). Developments in Photogrammetry. Invited paper of XXII ISPRS Congress in Malbourne

Zawieska D., Bakuła K., \& Podlasiak P. (2012). Analysis of surface deformation of construction element with usage of image-based matching. Archives of Photogrammetry, Cartography and Remote Sensing, 23, pp. 521-529

Zawieska, D. (2012). Multi-image Matching of Close Range Photographs for Photorealistic 3D Models Reconstruction. Warsaw: Warsaw University of Technology Publishing House

\section{Authors:}

Prof. Aleksandra Bujakiewicz ${ }^{1)}$, abujak7@wp.pl

Msc Jakub Markiewicz ${ }^{2)}$, j.markiewicz@gik.pw.edu.pl

Msc Krzysztof Bakuła ${ }^{2)}$, k.bakula@gik.pw.edu.pl

PhD Dorota Zawierska ${ }^{2)}$, d.zawieska@gik.pw.edu.pl

1) Koszalin University of Technology, Faculty of Civil Engineering, Environmental and Geodetic Sciences, ul. Śniadeckich 2, 75-453 Koszalin, Poland

2) Warsaw University of Technology, Faculty of Geodesy and Cartography, pl. Politechniki 1, 00-661 Warsaw, Poland 